DIW BERLIN

Discussion Papers

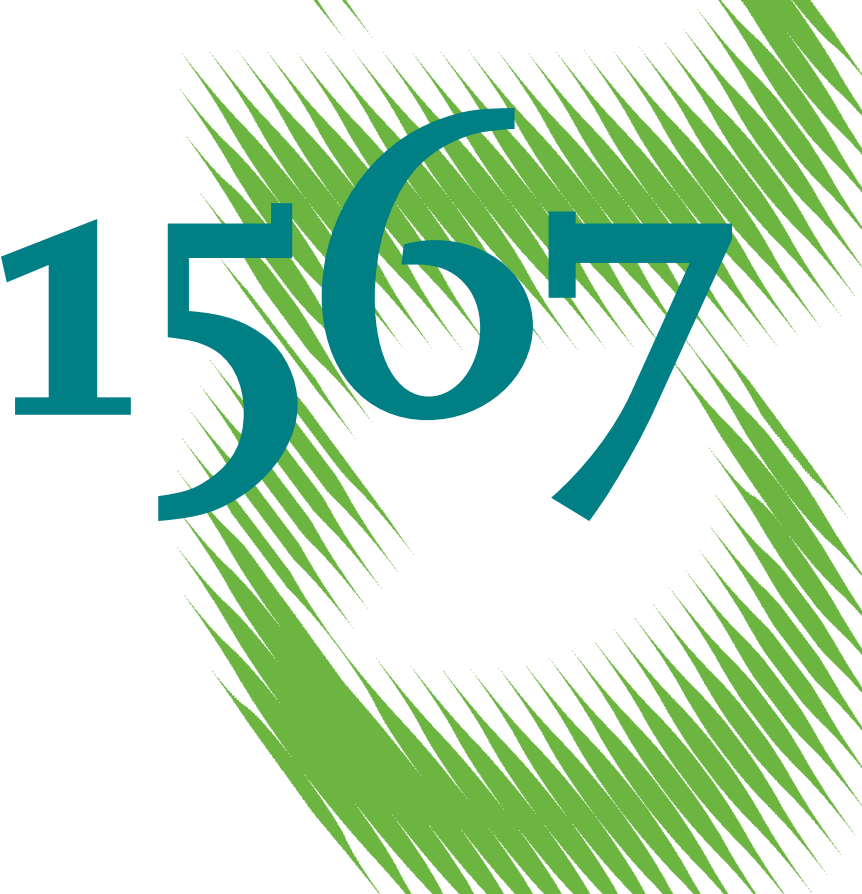

Price-Based Unit Commitment Electricity Storage Arbitrage with Piecewise Linear Price-Effects 
Opinions expressed in this paper are those of the author(s) and do not necessarily reflect views of the institute.

IMPRESSUM

(C) DIW Berlin, 2016

DIW Berlin

German Institute for Economic Research

Mohrenstr. 58

10117 Berlin

Tel. +49 (30) $89789-0$

Fax +49 (30) $89789-200$

http://www.diw.de

ISSN electronic edition 1619-4535

Papers can be downloaded free of charge from the DIW Berlin website:

http://www.diw.de/discussionpapers

Discussion Papers of DIW Berlin are indexed in RePEc and SSRN:

http://ideas.repec.org/s/diw/diwwpp.html

http://www.ssrn.com/link/DIW-Berlin-German-Inst-Econ-Res.html 


\title{
Price-Based Unit Commitment Electricity Storage Arbitrage with Piecewise Linear Price-Effects*
}

\author{
Tom Brijs ${ }^{\mathrm{a}, \mathrm{b}}$, Frederik Geth ${ }^{\mathrm{a}}$, Sauleh Siddiqui ${ }^{\mathrm{b}, \mathrm{d}, \mathrm{e}}$, \\ Benjamin F. Hobbs ${ }^{c}$, Ronnie Belmans ${ }^{\mathrm{a}}$
}

March 24, 2016

Electricity storage plants can be used for many applications, with one of the most studied applications being arbitrage in the day-ahead market. Although the arbitrage value is related to the presence of price spreads, it also depends on the effect of (dis)charge actions on prices, as arbitrage generally reduces price spreads by increasing off-peak prices when charging and decreasing peak prices when discharging. As such, there are two important assumptions in price-based unit commitment arbitrage models: first, whether the storage operator is assumed to have perfect knowledge of future prices, and second, whether they recognize that their (dis)charge actions may affect those prices, i.e., the price-taking or pricemaking assumption. This article proposes a comprehensive formulation of the arbitrage problem including detailed operating constraints, and focuses on relaxing the price-taking assumption by considering real-world price-effect data, published in the form of hourly piecewise linear relationships between quantity and price based on submitted bids, which are referred to as "market resilience functions". These can be used to (1) evaluate the price-taking and price-making assumptions based on simplified price-effects, and to (2) provide an upper limit to the arbitrage value under the assumption that prices and price-effects are known at the decision stage. In addition, a stepwise approximation to the piecewise linear functions is developed to reduce computation time, i.e., from mixed-integer nonconvex quadratic programming to mixed-integer linear programming, while providing lower- and upper bound approximations to the arbitrage value. The developed models are applied to the Belgian day-ahead market for 2014, and show that the price-effect has a strong impact on the operation and arbitrage value of large-scale storage.

Keywords: electricity storage, arbitrage, day-ahead market, price-effect, piecewise linear market resilience functions, price-based unit commitment.

* Submitted to the Journal of Energy Storage.

a Department of Electrical Engineering, University of Leuven (KU Leuven), Heverlee, Belgium, and EnergyVille Research Institute, Genk, Belgium.

b Department of Civil Engineering, The Johns Hopkins University, Baltimore, MD, USA.

c Department of Geography and Environmental Engineering and the Environment, Energy, Sustainability \& Health Institute, The Johns Hopkins University, Baltimore, MD, USA. 
d Department of Applied Mathematics and Statistics, The Johns Hopkins University, Baltimore, MD, USA.

e German Institute for Economic Research (DIW Berlin), Berlin, Germany.

E-mail: tom.brijs@esat.kuleuven.be, frederik.geth@esat.kuleuven.be, siddiqui@jhu.edu, bhobbs@jhu.edu, ronnie.belmans@esat.kuleuven.be. 


\section{Introduction}

\subsection{Motivation}

The storage of electricity represents a combination of three functions [1]: consuming electricity, accumulating the energy in some form, and generating electricity. Only part of the consumed electric energy is converted to energy stored in the buffer during charging because of a charge efficiency $0<\eta^{\mathrm{c}} \leq 1$, while only part of the stored energy is converted back into electric energy during discharging because of a discharge efficiency $0<\eta^{\mathrm{d}} \leq 1$. The buffered energy may also increase and decrease independent of the grid through exogenous power flows $p_{t}^{+} \geq 0$ (addition) and $p_{t}^{-} \geq 0$ (removal), e.g., water inflow and evaporation in the upper reservoir for pumped-hydro storage (PHS) plants. The general power balance of storage plants that consume electric power $p_{t}^{\mathrm{c}} \geq 0$ and generate electric power $p_{t}^{\mathrm{d}} \geq 0$, and store it in an energy buffer $e_{t} \geq 0$, is then:

$$
\underbrace{\frac{d e_{t}}{d t}}_{\triangle \text { Energy buffer }}=\underbrace{p_{\text {Addition }}^{\mathrm{c}} \cdot \eta^{\mathrm{c}}}_{\text {Electric origin }}-\underbrace{p_{t}^{\mathrm{d}} / \eta^{\mathrm{d}}}_{\text {Removal }}+\underbrace{\underbrace{p_{t}^{+}}_{\text {Addition }}-\underbrace{p_{t}^{-}}_{\text {Removal }}}_{\text {Exogenous origin }} .
$$

In recent years there has been a renewed interest in electricity storage due to the liberalization of electricity markets and the integration of variable renewable energy sources (RES). Their expected and unexpected variability results in an increased need for flexibility, which is the ability to provide power adjustments to deal with short-term variations $[2,3]$. Electricity storage plants can provide this flexibility by charging and discharging through interaction with an energy buffer. However, flexibility can also be provided by flexible generation and consumption, but also by the electric grid through which flexible capacity in neighboring regions can be accessed (Fig. 1). Market participants are only incentivized to integrate new flexible resources when the investment is profitable. Although electricity storage plants can be used for many applications (e.g., arbitrage, portfolio optimization, frequency control, voltage support, black-start service $[4,5]$ ) and maximizing the value of storage requires the aggregation of different applications, one of the most studied and well-known applications is arbitraging day-ahead (DA) market electricity prices $[6,7]$. This article focuses on the arbitrage application as the sole revenue source.

\subsection{Scope and approach}

Classic definitions of arbitrage denote making a riskless profit by simultaneously buying and selling a similar commodity with net zero investment. However, in a broader context any activity in which a player buys a commodity at a relatively low price and sells a similar commodity, or commodity in which the former can be converted, at a relatively high price for profit can be referred to as arbitrage. This broader definition allows to include initial investments, does not require simultaneity of the purchase and sale, and furthermore does not require a single commodity either (i.e., so-called cross-commodity arbitrage) [8]. In the context of this article, arbitrage is defined as the capturing of price spreads over time in a single market, being the DA market, by means of electricity storage plants. Although the arbitrage value is directly related to the presence of these price spreads, it also depends on the price-effect of (dis)charge actions, as additional storage capacity generally reduces price spreads by increasing off-peak prices when charging as well as decreasing on-peak prices when discharging.

In contrast to cost-based unit commitment (UC), which refers to the scheduling of generation capacity to meet system load at minimum cost, the scheduling of units 


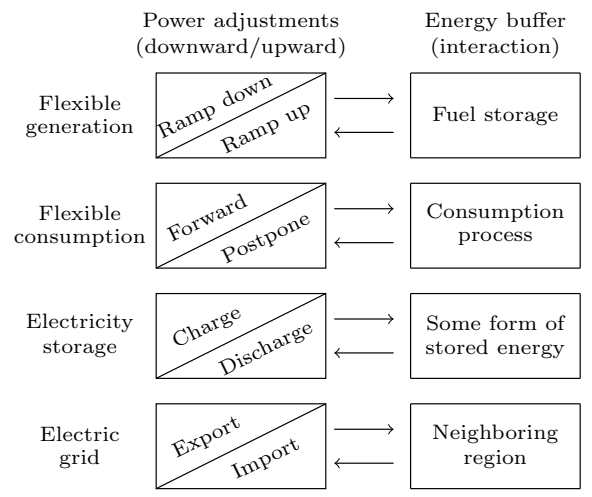

Figure 1: Overview of power system flexibility sources.

with the objective to maximize profit based on price signals is referred to as pricebased unit commitment (PBUC) [9]. The arbitrage application is widely discussed in the literature, both from a system (e.g., [10-15]) and from an individual storage plant's PBUC perspective, the latter being the focus of this article. Generally, there are two important assumptions in PBUC arbitrage models: the first is related to the storage operator's assumed knowledge of future prices, i.e., the (im)perfect price foresight assumption, while the second is related to whether they recognize that their (dis)charge actions may affect those prices, i.e., the price-taking or price-making assumption $[16,17]$. A large share of the existing PBUC work assumes perfect foresight of future prices and the storage plant to be small enough to be a price-taker in the considered market (e.g., [18-23]). Although quite some studies discuss a relaxation of the perfect price foresight assumption (e.g., [16, 24-30]), less attention has been given to the relaxation of the price-taking assumption in PBUC arbitrage models. However, either large-scale or multiple small-scale storage plants that are operated cooperatively could benefit from considering the price-effect of (dis)charge actions. Even when deciding on the (dis)charge schedule as a price-taker, considering the price-effect in the ex-post calculation of the realized profit is important for owners of large storage capacities as arbitrage may reduce effective price spreads.

First, $[16,31]$ introduce a method to account for this price-effect based on an observed linear relationship between the system load and price. Second, [32] introduces a constant so-called market resilience factor to represent the price-effect of (dis)charge actions. Third, [33] and [34] propose methodologies to relax the price-taking assumption by taking into account the residual inverse demand function. Although these methodologies provide insight in the arbitrage value and operation of large storage capacities, due to a lack of market data or a different research scope they are based on rather conceptual and simplified price-effects and therefore result in (1) a suboptimal (dis)charge schedule and accompanying arbitrage value with respect to the actual price-effect, and (2) an ex-post gap between the expected and realized profit.

Therefore, this article focuses on relaxing the price-taking assumption by including real-world market resilience data, which illustrates the impact on the DA price of a change in offer or demand volume for each hour, published by several European power exchanges. ${ }^{1}$ This data represents the most detailed available price-effect data, as it is obtained by the power exchange running the market-clearing algorithm again for alternative scenarios, and thus takes into account (1) the hourly aggregated supply

\footnotetext{
${ }^{1}$ In contrast to the considered market resilience data, the price elasticity of demand refers to the relative change in demand as a result from a relative change in the price, and is typically negative as the demand for most commodities decreases as the price increases [35].
} 
and demand curves, (2) interaction with neigboring markets through market-coupling, and (3) the presence of complex orders. This article focuses on the arbitrage value of additional storage capacity in the DA market, but does not aim to provide bidding strategies for storage plants (e.g., [36]). Instead, the storage operator is assumed to self-schedule its (dis)charge actions against a set of DA prices and market resilience functions that reflect how price reacts to changes in quantity.

\subsection{Contributions}

The main contributions of this article with respect to previous research on electricity storage arbitrage is a comprehensive formulation of the electricity storage arbitrage problem including detailed operating constraints, and the presentation of a new methodology to account for the price-effect of (dis)charge actions. Since the latter is based on the implementation of real-world market resilience functions in the PBUC arbitrage model, it includes the most detailed available price-effect data. As such, this article presents a measure to (1) evaluate the performance of the price-taking assumption and price-making assumptions based on more conceptual and simplified price-effects, and to (2) provide an upper limit to the arbitrage value, given current market conditions, if both the hourly prices and price-effects are assumed to be known at the decision stage. The former is done for a storage operator that assumes to be a price-taker in the market by using the price-effect data to ex-post calculate the realized profit, as opposed to the expected profit based on prices that would occur in the absence of (dis)charge actions. In addition, as the piecewise linear nature of the market resilience data poses computational challenges, a stepwise approximation of the piecewise linear functions is proposed which reduces computational effort significantly while providing lower- and upper bound approximations to the piecewise linear results. The analyses are executed for the Belgian DA market, and show that the price-effect has a strong impact on the operation and arbitrage value of large-scale storage.

This article is structured as follows. Section 2 discusses the price-effect in PBUC formulations of the storage arbitrage problem. Next, Section 3 provides a comprehensive formulation of the arbitrage problem based on a price-taking assumption, while Section 4 extends this formulation by including the price-effect through piecewise linear and stepwise approximated market resilience functions. Section 5 discusses the results. Finally, Section 6 provides conclusions.

\section{The price-effect of storage actions}

\subsection{Literature review}

Although a relaxation of the price-taking assumption (Fig. 2a) has been studied extensively in other frameworks (e.g., equilibrium models [37-39]), it has only been studied to a limited extent in PBUC electricity storage arbitrage models. First, references $[16,31]$ assume a monthly linear relationship between the price and system load for the DA market in the United States' PJM region, obtained by using ordinary least squares regression. The slope associated with the OLS function is assumed to be non-decreasing and to capture the price-effect of (dis)charge actions (Fig. 2b). Second, [32] considers the Belgian DA market and defines a constant market resilience factor as price-effect for all (dis)charge volumes for the entire year (Fig. 2b). This factor is based on the DA market resilience data discussed in Section 1, but does not consider its time-varying and piecewise linear nature. Third, two approaches include the price-effect by considering residual inverse demand curves. In [33] the price-effect is studied for the DA Iberian Electricity Market MIBEL, and is defined by a residual inverse demand curve that depends on the slopes of the demand and supply curves, and which is modeled through an approximated sigmoid function. Contrarily, [34] 


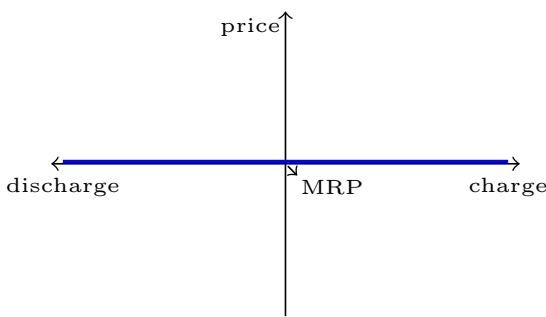

(a) no price-effect (price-taker)

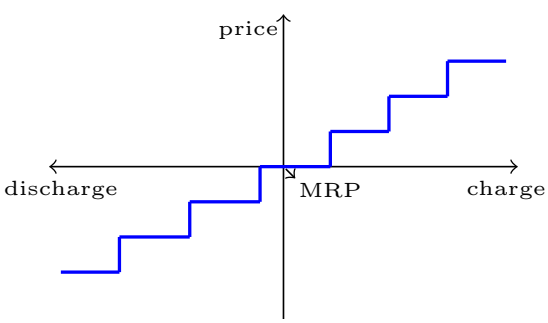

(c) stepwise price-effect

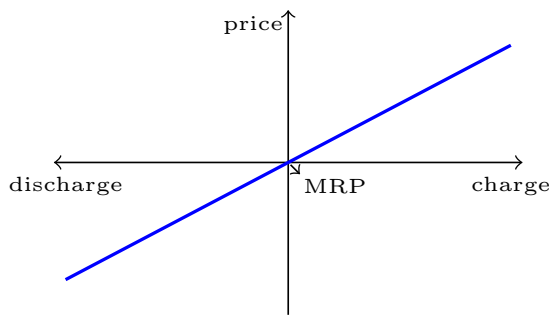

(b) linear price-effect

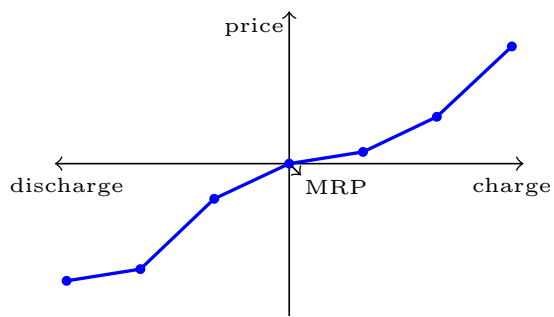

(d) piecewise linear price-effect

Figure 2: Overview of the price-taking vs. different price-making assumptions. The market reference point (MRP) refers to the situation without participation of the additional storage capacity, and is located at the origin.

studies the price-effect in the context of the Greek DA market. The demand curve is assumed to be perfectly inelastic, while the mirrored image of the stepwise supply curve from other generation is assumed to represent the residual inverse demand curve. The resulting price is determined by the intersection of the stepwise supply curve and the vertical demand curve, with the latter's position depending on the storage plant's (dis)charge actions (Fig. 2c).

When considering generation capacity, a similar approach to [34] is applied in [40, 41] for a conventional hydro power plant. In addition, [42] discusses a methodology for generation companies to provide hourly offers by considering a series of possible residual inverse demand curves. These are selected from recent days similar to the considered day in terms of hourly demand, and are thus based on historical offers submitted by competing players.

Due to either a lack of available market data or the focus on a different research scope, the existing approaches include simplified representations of the price-effect. This may result in suboptimal (dis)charge schedules and resulting arbitrage value when evaluating against the actual price-effect, and incorrect estimates of the realized profit. When calculating the change in price, inaccuracies may originate from not considering (1) the time-varying aggregated supply and demand curves, (2) the acceptance of new block orders and rejection of currently cleared ones, and (3) changes in cross-border flows due to the coupling of geographically adjacent markets.

\subsection{Hourly piecewise linear market resilience functions}

The degree to which additional demand and supply would affect the Belgian DA price is captured by the market resilience data, published in the form of hourly piecewise linear functions (Fig. 2d) by the Belpex power exchange [43]. ${ }^{2}$ As stated before, this data is obtained by rerunning the market-clearing algorithm for six different scenarios

\footnotetext{
${ }^{2}$ Such market resilience data is also published by the APX [44] and EPEX SPOT [45] power exchanges for other European countries.
} 
(i.e., $50 \mathrm{MWh}, 250 \mathrm{MWh}, 500 \mathrm{MWh}$ additional offer or demand volume at any price), and takes into account the aggregated supply and demand curves, interaction with neighboring markets through market-coupling, and presence of complex orders (e.g., block orders). Contrarily, only using the published aggregated offer curves from within a single market omits changes in cross-border flows due to market-coupling as well as the change in clearing of complex orders for the following reasons:

- The Belpex DA market is coupled with other European power exchanges. The market-clearing algorithm combines the supply and demand bid information of the different exchanges to optimize the utilization of the available interconnection capacity. In case of a price difference between geographical markets, electric energy is exchanged until the price difference is eliminated or all available interconnection capacity is used. Consequently the effect of a local increase in offer or demand may not only affect the local market price but also the price in the coupled markets. ${ }^{3}$ Since the price-effect in one market is a function of the market resilience of the local market as well as of the coupled markets, and the available interconnection capacity, merely considering the local power exchange's aggregated curves results in an overestimation of the total price-effect, including the impact of changed imports and exports.

- The standardized orders in DA markets are limit orders, i.e., hourly offered or requested quantities with a certain price limit. Besides these hourly orders, most exchanges also allow other, more complex, orders [46]. The most common one is a block order, which consists of quantities that are offered or requested in multiple hours at an average price limit and which has to be accepted completely or not at all. Due to their specific nature, such accepted sale and buy block orders are introduced in the aggregated supply and demand curve at the minimum and maximum price, respectively, thereby ignoring their price-sensitive character. If the price-effect would be simulated by simply shifting aggregated supply and demand curves, this would not capture the extent to which initially rejected block orders may be accepted and accepted block orders may now be rejected.

Finally, it is important to note that PBUC formulations of the arbitrage problem assume that other players will not change their behavior with participation of the additional storage capacity. PBUC models considering a price that is determined exogenously, i.e., independent of the considered storage plant's (dis)charge actions, assume a price-taking assumption and are useful to represent perfect competition conditions. In contrast, PBUC models in which a storage operator maximizes profit while considering its price-effect given the decisions of the competing players represent so-called leader-in-price models $[47,48]$. In more complex games other players might react and change their behavior in response to entry of additional storage capacity.

\section{Day-ahead electricity storage arbitrage}

The continuous time dimension, represented by time index $t$, is discretized, with $h$ representing the discrete index, while the model formulations assume a fixed time step length $T^{\mathrm{h}}$ of one hour (Fig. 3). The storage operator uses electricity storage resources to maximize the arbitrage value on a daily basis over an optimization horizon of 48 hourly time steps $(\forall h \in \mathbb{H})$. In order to ensure that energy stored at the end of each 24 hour optimization period has so-called carryover value [16], each optimization is done with a 48 hour horizon to determine the dispatch of each 24 hour period. The

\footnotetext{
${ }^{3}$ Since a demand increase in a market would result in an increased local price, this leads to an increased import (if interconnection capacity is available) which in turn increases the price in the exporting market and (partially) offsets the price increase in the local market. A similar reasoning holds for a supply increase.
} 

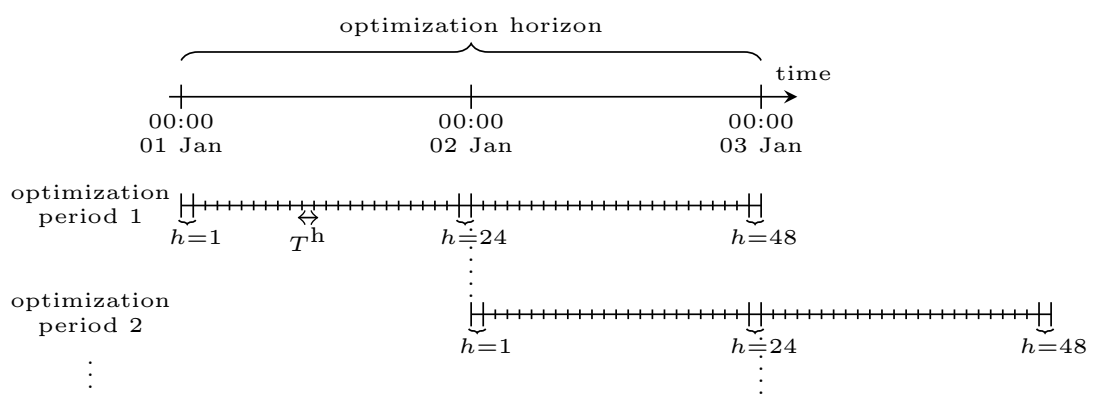

Figure 3: Illustration of the time dimension discretization and rolling optimization horizon.

storage operator is thus assumed to decide upon the charge power $p_{h}^{\mathrm{c}}$ and discharge $p_{h}^{\mathrm{d}}$ power based on short-term DA price differences throughout the day, and is assumed to have a perfect knowledge of these prices for the upcoming optimization period. ${ }^{4}$

An electricity storage plant can typically be characterized by the minimum and maximum charge power rating $P^{\mathrm{c}, \text { min }}$ and $P^{\mathrm{c}, \max }$, discharge power rating $P^{\mathrm{d}, \text { min }}$ and $P^{\mathrm{d} \text {,max }}$, and energy storage capacity $E^{\mathrm{min}}$ and $E^{\mathrm{max}}$, the charge and discharge efficiency $\eta^{\mathrm{c}}$ and $\eta^{\mathrm{d}}$, and the down- and upward ramp rate in charge mode $R^{\mathrm{c} \text {,do }}$ and $R^{\text {c,up }}$ and in discharge mode $R^{\mathrm{d} \text {,do }}$ and $R^{\mathrm{d} \text {,up }}$. In addition, storage plants have a limited lifetime, which is either determined by the calendar life $N^{\text {cal }}$ in case of infrequent use or by the cycle-life $N^{\text {cyc }}$ in case of frequent use [49]. ${ }^{5}$ The calendar life is the maximum time that the storage plant can be used, independent from the use, while the cycle-life takes into account the deterioration of the energy storage subsystem due to use. The latter is particularly important when considering a battery energy storage system (BESS) due to the partial nonreversibility of the chemical reactions. Although there is no direct constraint on the number of cycles during each optimization period, due to the limited cycle-life it is implied that the targeted cycling rate $N^{\text {cyc }} / N^{\text {cal }}$ is constant throughout the lifetime. If the cycling rate $n^{\text {cyc }}(9)$ is lower than or equal to the targeted cycling rate, the depreciation cost resulting from cycling $c^{\text {cyc }}$ is zero, otherwise $c^{\text {cyc }}>0$ (Fig. 4). This formulation to include the limited cycle-life and resulting depreciation cost is derived from [49]. Contrary to BESS, for PHS plants the cycle-life is sufficiently large such that $c^{\text {cyc }}$ is negligible. Furthermore, this article assumes changes in the buffered energy due to exogenous power flows to be negligible in the short-term. ${ }^{6}$

Since the storage operator is assumed to be a price-taker in this initial problem formulation, the (dis)charge schedule is optimized not taking into account the impact on the price without its participation $\lambda_{h}^{\mathrm{da}, \mathrm{o}}$. The resulting problem is a mixed-integer linear program (MILP) which is solved in GAMS using the CPLEX solver [50]:

\footnotetext{
${ }^{4}$ The rolling optimization horizon restricts the perfect foresight to the next 48 hours.

${ }^{5}$ The cycle-life is usually defined as the number of cycles before the remaining usable capacity falls below $80 \%$ of the initial storage capacity due to wear.

${ }^{6}$ Table 1 provides an overview of the symbols for sets, decision variables, and parameters. While formulas are provided assuming SI units (or other base units), input data and results are provided in commonly used units in electrical engineering, e.g., MWh instead of J.
} 


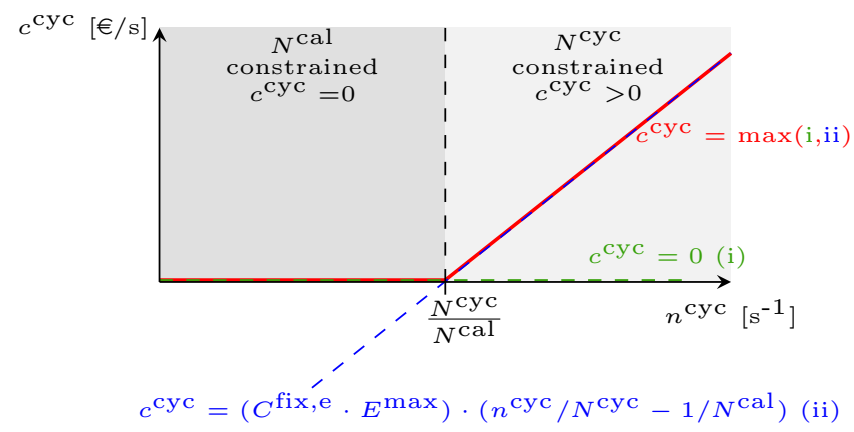

Figure 4: Illustration of the opportunity cost for wearing out the energy storage subsystem.

$$
\begin{array}{rlrl}
\pi^{\mathrm{op}}= & \max _{\substack{b_{h}, c^{\mathrm{cyc}}, e_{h}, n^{\mathrm{cyc}}, p_{h}^{\mathrm{c}}, p_{h}^{\mathrm{d}}}} \sum_{h \in \mathbb{H}} \lambda_{h}^{\mathrm{da}, \mathrm{o}} \cdot\left[T^{\mathrm{h}} \cdot\left(p_{h}^{\mathrm{d}}-p_{h}^{\mathrm{c}}\right)\right] /\left(|\mathbb{H}| \cdot T^{\mathrm{h}}\right)-c^{\mathrm{cyc}}, \\
\text { s.t. } & e_{h}=e_{h-1}+T^{\mathrm{h}} \cdot\left(p_{h}^{\mathrm{c}} \cdot \eta^{\mathrm{c}}-p_{h}^{\mathrm{d}} / \eta^{\mathrm{d}}\right), & & \forall h \in \mathbb{H}, \\
& -R^{\mathrm{c}, \mathrm{do}} \cdot P^{\mathrm{c}, \max } \leq\left(p_{h}^{\mathrm{c}}-p_{h-1}^{\mathrm{c}}\right) / T^{\mathrm{h}} \leq R^{\mathrm{c}, \mathrm{up}} \cdot P^{\mathrm{c}, \mathrm{max}}, & \forall h \in \mathbb{H}, \\
& -R^{\mathrm{d}, \mathrm{do}} \cdot P^{\mathrm{d}, \max } \leq\left(p_{h}^{\mathrm{d}}-p_{h-1}^{\mathrm{d}}\right) / T^{\mathrm{h}} \leq R^{\mathrm{d}, \mathrm{up}} \cdot P^{\mathrm{d}, \max }, & \forall h \in \mathbb{H}, \\
& 0 \leq P^{\mathrm{c}, \min } \cdot b_{h} \leq p_{h}^{\mathrm{c}} \leq P^{\mathrm{c}, \max } \cdot b_{h}, & & \forall h \in \mathbb{H}, \\
& 0 \leq P^{\mathrm{d}, \min } \cdot\left(1-b_{h} \leq p_{h}^{\mathrm{d}} \leq P^{\mathrm{d}, \max } \cdot\left(1-b_{h}\right),\right. & & \forall h \in \mathbb{H}, \\
& 0 \leq E^{\mathrm{min}} \leq e_{h} \leq E^{\max }, & & \\
& n^{\mathrm{cyc}}=\eta^{\mathrm{c}} \cdot \sum_{h \in \mathbb{H}} p_{h}^{\mathrm{c}} / E^{\max }, & \\
& c^{\mathrm{cyc}} \geq\left(C^{\mathrm{fix}, \mathrm{e}} \cdot E^{\max }\right) \cdot\left(n^{\mathrm{cyc}} / N^{\mathrm{cyc}}-1 / N^{\mathrm{cal}}\right), & \\
& c^{\mathrm{cyc}}, e_{h}, n^{\mathrm{cyc}}, p_{h}^{\mathrm{c}}, p_{h}^{\mathrm{d}} \in \mathbb{R}_{+}, b_{h} \in\{0,1\}, \mathbb{H} \subset \mathbb{N}, & & \forall h \in \mathbb{H} .
\end{array}
$$

The objective value in (2) expresses the operating profit $\pi^{\mathrm{op}}$, which does not consider the electricity storage plant's investment cost. Constraint (3) expresses the intertemporal character of electricity storage, while (4) and (5) limit the change in (dis)charge power by the storage plant's ramp rates. Constraints (6) - (8) represent capacity bounds on the (dis)charge power and storage capacity, with binary variable $b_{h}$ ensuring that the storage plant is operated with a strict separation of the electricity consumption and generation phase, i.e., $p_{h}^{\mathrm{c}} \cdot p_{h}^{\mathrm{d}}=0 \forall h$. If however simultaneous charging and discharging is technically feasible (e.g., in certain pumped-hydro storage (PHS) plants), it is profitable to ignore the nonsimultaneity constraint during negative price periods. $^{7}$ The nonnegativity of $c^{\text {cyc }}$ and (9) - (10) represent the convex relaxation $^{8}$ of the max operator illustrated in Fig. 4, with $C^{\text {fix,e }}$ the investment cost of the energy-component of the storage plant. The intertemporal equation (3) indicates that if the storage plant consumes $p_{h}^{\mathrm{c}}$ electric power during $T^{\mathrm{h}}$ then the stored energy level $e_{h}$ increases by $T^{\mathrm{h}} \cdot p_{h}^{\mathrm{c}} \cdot \eta^{\mathrm{c}}$, while if the storage plant generates $p_{h}^{\mathrm{d}}$ electric power during $T^{\mathrm{h}}$ then the stored energy level $e_{h}$ decreases by $T^{\mathrm{h}} \cdot p_{h}^{\mathrm{d}} / \eta^{\mathrm{d}}$. When $h=1$ in (3) - (5), index $h-1$ indicates $h=24$ of the previous daily optimization period, except for the

\footnotetext{
${ }^{7}$ During negative price periods the storage operator is paid to consume electric energy, and will therefore attempt to fill the storage buffer as quickly as possible. When the upper limit of the storage buffer is reached during these periods, it is profitable to charge and discharge simultaneously, thereby being remunerated for the incurred efficiency losses.

${ }^{8} \mathrm{As} c^{\mathrm{cyc}}$ is minized in (2), the convex relaxation leads to a result satisfying the original max operator.
} 
Table 1: Table of symbols.

\begin{tabular}{|c|c|c|c|c|}
\hline Type & Symbol & Quantity & Unit (SI) & Typical unit \\
\hline \multirow[t]{4}{*}{ Sets } & $h \in \mathbb{H}$ & Time steps & - & - \\
\hline & $k \in \mathbb{K}$ & Piecewise breakpoints & - & - \\
\hline & $t \in \mathbb{T}$ & Time stamps & - & - \\
\hline & $u \in \mathbb{U}$ & Stepwise steps & - & - \\
\hline \multirow[t]{12}{*}{ Variables } & $b_{h}$ & Binary variable & - & - \\
\hline & $c^{\mathrm{cyc}}$ & Depreciation cost & $€ / \mathrm{s}$ & $€ /$ year \\
\hline & $e_{h}, e_{t}$ & Stored energy & $\mathrm{J}$ & MWh \\
\hline & $n^{\text {cyc }}$ & Cycling rate & $\mathrm{s}^{-1}$ & year $^{-1}$ \\
\hline & $p_{h}^{\mathrm{c}}, p_{h, u}^{\mathrm{c}}, p_{t}^{\mathrm{c}}$ & Charge power & $\mathrm{W}$ & MW \\
\hline & $p_{h}^{\mathrm{d}}, p_{h, u}^{\mathrm{d}, u}, p_{t}^{\mathrm{d}}$ & Discharge power & W & MW \\
\hline & $p_{t}^{+}, p_{t}^{-}$ & Exogenous power flows & $\mathrm{W}$ & MW \\
\hline & $r_{h, k}$ & Binary variable & - & - \\
\hline & $s_{h, u}$ & Binary variable & - & - \\
\hline & $\delta_{h, k}$ & SOS2 variable & - & - \\
\hline & $\lambda_{h}^{\text {da }}$ & Day-ahead price & $€ / J$ & $€ / \mathrm{MWh}$ \\
\hline & $\pi^{n}$ & Operating profit & $€ / \mathrm{s}$ & $€ /$ year \\
\hline \multirow[t]{17}{*}{ Parameters } & $C^{\mathrm{fix}, \mathrm{e}}$ & Energy-component investment cost & $€ / J$ & $€ / \mathrm{kWh}$ \\
\hline & $E^{\max }, E^{\min }$ & Storage capacity bounds & $\mathrm{J}$ & MWh \\
\hline & $N^{\text {cal }}$ & Calendar life & $\mathrm{s}$ & year \\
\hline & $N^{\text {cyc }}$ & Cycle-life & - & - \\
\hline & $N_{h, k}^{\mathrm{sw}}$ & Number of steps & - & - \\
\hline & $P^{\mathrm{c}, \max }, P^{\mathrm{c}, \min }$ & Charge power rating bounds & W & MW \\
\hline & $P^{\mathrm{d}, \max }, P^{\mathrm{d}, \min }$ & Discharge power rating bounds & $\mathrm{W}$ & MW \\
\hline & $R^{\mathrm{c}, \mathrm{do}}, R^{\mathrm{d}, \mathrm{do}}$ & Downward ramp rate & $\% / \mathrm{s}$ & $\% / \min$ \\
\hline & $R^{\text {c,up }}, R^{\text {d,up }}$ & Upward ramp rate & $\% / \mathrm{s}$ & $\% / \min$ \\
\hline & $R_{h, k}^{\text {div }}$ & Remainder & - & - \\
\hline & $S^{\text {tar }}$ & Target step height & $€ / J$ & $€ / \mathrm{MWh}$ \\
\hline & $S_{h, k}^{\text {upd }}$ & Updated step height & $€ / J$ & $€ / \mathrm{MWh}$ \\
\hline & $T^{n, \mathrm{~h}}$ & Time step length & s & $\mathrm{h}$ \\
\hline & $X_{k}^{\mathrm{pw}}, X_{h, u}^{\mathrm{sw}, \mathrm{lo}}, X_{h, u}^{\mathrm{sw}, \mathrm{up}}$ & (Dis)charge volume & $\mathrm{J}$ & MWh \\
\hline & $Y_{h, k}^{\mathrm{pw}, u}, Y_{h, u}^{\mathrm{sw}}$ & Day-ahead price & $€ / J$ & $€ / \mathrm{MWh}$ \\
\hline & $\eta^{\mathrm{c}}, \eta^{\mathrm{d}}$ & (Dis)charge efficiency & $\%$ & $\%$ \\
\hline & $\lambda_{h}^{\mathrm{da}, \mathrm{o}}$ & Initial day-ahead price & $€ / J$ & $€ / \mathrm{MWh}$ \\
\hline
\end{tabular}


first optimization period where index $h-1$ at $h=1$ refers to starting values for the (dis)charge power and stored energy.

Given the (dis)charge schedule decided upon by the storage operator that assumes to be a price-taker in the market, the resulting DA prices after participation can be calculated ex-post by interpolating the piecewise linear market resilience functions. These prices are used to calculate the realized $\pi^{\mathrm{op}}$ as opposed to the expected $\pi^{\mathrm{op}}$ following $\lambda_{h}^{\mathrm{da}, \mathrm{o}}$.

\section{Relaxing the price-taking assumption}

\subsection{Including time-varying piecewise-linear market resilience func- tions}

Detailed price resilience data is provided by several European power exchanges in the form of time-varying piecewise linear functions (Fig. 5), which may be nonconvex and include both increasing and counterintuitive decreasing linear segments. Segments are considered to be intuitive if the price decreases with additional supply and increases with additional demand, while counterintuitive segments are the result of differences in accepted block orders: additional supply can cause supply (demand) block orders that are accepted (rejected) in the reference case to become rejected (accepted), while additional demand can cause demand (supply) block orders that are accepted (rejected) in the reference case to become rejected (accepted). For the Belgian DA market in 2014, $70.5 \%$ of the segments include intuitive slopes, $19.5 \%$ include counterintuitive slopes, and $10 \%$ of the segments are horizontal, i.e., no change in price due to a change in quantity.

To include these piecewise linear functions in the arbitrage problem formulation, a set $k \in \mathbb{K}$ of piecewise linear function breakpoints and a variable $\delta_{h, k}$, which can be considered as a Special Ordered Set of type two (SOS2) variable [51], are introduced. For the Belgian DA market, the piecewise linear functions have seven fixed breakpoints (i.e., $|\mathbb{K}|=7$ ) along the $\mathrm{x}$-axis (i.e., (dis)charge volumes) indicated by $X_{k}^{\mathrm{pw}}$, while the corresponding time-dependent y-axis values (i.e., prices) are indicated by $Y_{h, k}^{\mathrm{pw}}$. The MRP lies at breakpoint $k=4$, with $X_{4}^{\mathrm{pw}}=0$ and $Y_{h, 4}^{\mathrm{pw}}=\lambda_{h}^{\mathrm{da}, \mathrm{o}}$. For each breakpoint $k$ there is a nonnegative $\delta_{h, k}$, which is bounded by 1 and which may be greater than 0 for at most two breakpoints. If there are two positive $\delta_{h, k}$, they must correspond to adjacent breakpoints and take on values between 0 and 1, depending the weighted share of the corresponding breakpoints' $\mathrm{x}$-axis value and $\mathrm{y}$-axis value in the calculation of the chosen (dis)charge volume and resulting DA price:

$$
\begin{aligned}
& \delta_{h, k}=\frac{\left|X_{k+1}^{\mathrm{pw}}-T^{\mathrm{h}} \cdot\left(p_{h}^{\mathrm{d}}-p_{h}^{\mathrm{c}}\right)\right|}{\left|X_{k}^{\mathrm{pw}}-X_{k+1}^{\mathrm{pw}}\right|}=\frac{\left|Y_{h, k+1}^{\mathrm{pw}}-\lambda_{h}^{\mathrm{da}}\right|}{\left|Y_{h, k}^{\mathrm{pw}}-Y_{h, k+1}^{\mathrm{pw}}\right|}, \\
& \delta_{h, k+1}=\frac{\left|X_{k}^{\mathrm{pw}}-T^{\mathrm{h}} \cdot\left(p_{h}^{\mathrm{d}}-p_{h}^{\mathrm{c}}\right)\right|}{\left|X_{k}^{\mathrm{pw}}-X_{k+1}^{\mathrm{pw}}\right|}=\frac{\left|Y_{h, k}^{\mathrm{pw}}-\lambda_{h}^{\mathrm{da}}\right|}{\left|Y_{h, k}^{\mathrm{pw}}-Y_{h, k+1}^{\mathrm{pw}}\right|} .
\end{aligned}
$$

Alternatively, one $\delta_{h, k}$ might take on the value 1 , which means that the storage operator decides to be at one of the breakpoints. The adjacency condition is enforced by incorporating additional binary variables $r_{h, k}$, corresponding to the segments between adjacent breakpoints.

The DA arbitrage objective function is now (14), which is still subject to (3) - (11), and is now additionally subject to (15) - (20). The resulting problem is a mixed-integer nonconvex quadratic program (nonconvex MIQP) which is solved in GAMS using the SBB solver [52]: 


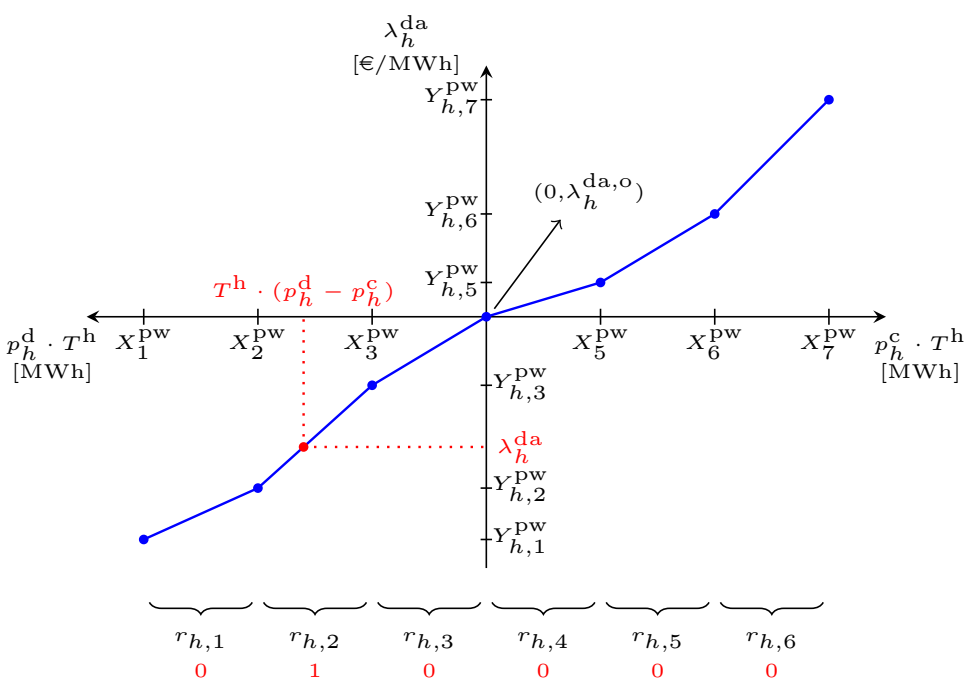

Figure 5: Illustration of piecewise linear market resilience functions.

$$
\pi^{\mathrm{op}}=\max _{\substack{b_{h}, c^{\mathrm{cyc}}, e_{h}, n^{\mathrm{cyc}}, p_{h}^{\mathrm{c}}, p_{h}^{\mathrm{d}}, r_{h, k}, \lambda_{h}^{\mathrm{da}}, \delta_{h, k}}} \sum_{h \in \mathbb{H}} \lambda_{h}^{\mathrm{da}} \cdot\left[T^{\mathrm{h}} \cdot\left(p_{h}^{\mathrm{d}}-p_{h}^{\mathrm{c}}\right)\right] /\left(|\mathbb{H}| \cdot T^{\mathrm{h}}\right)-c^{\mathrm{cyc}},
$$

s.t. $\quad(3)-(11)$,

$$
\begin{array}{ll}
T^{\mathrm{h}} \cdot\left(p_{h}^{\mathrm{d}}-p_{h}^{\mathrm{c}}\right)=\sum_{k \in \mathbb{K}} \delta_{h, k} \cdot X_{k}^{\mathrm{pw}}, & \forall h \in \mathbb{H}, \\
\lambda_{h}^{\mathrm{da}}=\sum_{k \in \mathbb{K}} \delta_{h, k} \cdot Y_{h, k}^{\mathrm{pw}}, & \forall h \in \mathbb{H}, \\
\sum_{k \in \mathbb{K}} \delta_{h, k}=1, & \forall h \in \mathbb{H}, \\
\sum_{k \in \mathbb{K} \backslash\{|\mathbb{K}|\}} r_{h, k}=1, & \forall h \in \mathbb{H}, \\
\delta_{h, k} \leq r_{h, k}, & \forall h \in \mathbb{H}, k=1, \\
\delta_{h, k} \leq r_{h, k-1}+r_{h, k}, & \forall h \in \mathbb{H}, k \in \mathbb{K} \backslash\{1,|\mathbb{K}|\}, \\
\delta_{h, k} \leq r_{h, k-1}, & \forall h \in \mathbb{H}, k=|\mathbb{K}|, \\
\delta_{h, k} \in \mathbb{R}_{+}, \lambda_{h}^{\mathrm{da}} \in \mathbb{R}, r_{h, k} \in\{0,1\}, \mathbb{K} \subset \mathbb{N}, & \forall h \in \mathbb{H}, k \in \mathbb{K} .
\end{array}
$$

Since solving this nonconvex MIQP requires significant computation time, a stepwise approximation of the piecewise linear functions is discussed in Section 4.2. This approximation converts the problem into an easier to solve MILP, and furthermore allows to determine lower- and upper bound approximations to the piecewise linear outcome.

\subsection{Stepwise approximation of time-varying piecewise linear func- tions}

This method approximates each linear segment of the piecewise linear market resilience functions by a stepwise function with identical step heights. A target step height $S^{\text {tar }}$ is set for all linear segments, but is updated to $S_{h, k}^{\text {upd }}$ in (22) by (23) for each time 
slot and linear segment individually if the divison's remainder $0 \leq R_{h, k}^{\mathrm{div}} \leq 1$ in (21) is greater than zero:

$$
\begin{array}{ll}
\frac{\left|Y_{h, k}^{\mathrm{pw}}-Y_{h, k+1}^{\mathrm{pw}}\right|}{S_{\mathrm{tar}}^{\mathrm{sw}}}=N_{h, k}^{\mathrm{sw}}+R_{h, k}^{\mathrm{div}}, & \forall h \in \mathbb{H}, k \in \mathbb{K} \backslash\{|\mathbb{K}|\}, \\
N_{h, k}^{\mathrm{sw}}=N_{h, k}^{\mathrm{sw}}+1 \quad \text { if } \quad R_{h, k}^{\mathrm{div}}>0, & \forall h \in \mathbb{H}, k \in \mathbb{K} \backslash\{|\mathbb{K}|\}, \\
S_{h, k}^{\mathrm{upd}}=\frac{\left|Y_{h, k}^{\mathrm{pw}}-Y_{h, k+1}^{\mathrm{pw}}\right|}{N_{h, k}^{\mathrm{sw}}}, & \forall h \in \mathbb{H}, k \in \mathbb{K} \backslash\{|\mathbb{K}|\},
\end{array}
$$

with $N_{h, k}^{\mathrm{sw}} \in \mathbb{N}_{0}$ the number of steps to approximate the linear segment between breakpoints $k$ and $k+1$. The resulting time-varying stepwise function's total number of steps $\sum_{k \in \mathbb{K} \backslash\{|\mathbb{K}|\}} N_{h, k}^{\mathrm{sw}}$, determined by $S^{\mathrm{tar}}$, relates to the preferred trade-off between computation time and approximation error. ${ }^{9}$

For each time slot, three stepwise functions are constructed. Two of them allow to calculate a lower- and upper bound to $\pi^{\text {op }}$ following the piecewise linear market resilience functions. The lower bound is calculated by considering a larger price-effect compared to the piecewise linear price-effect, thereby underestimating $\pi^{\mathrm{op}}$, while the upper bound is calculated by considering a smaller price-effect, thereby overestimating $\pi^{\mathrm{op}}$. The former is based on a stepwise function that approximates the piecewise linear function such that $\lambda_{h}^{\text {da }}$ is identical or less favorable for all (dis)charge actions, while the latter's stepwise function approximates the piecewise linear function such that $\lambda_{h}^{\mathrm{da}}$ is identical or more favorable (Fig. 6). ${ }^{10}$ Although this method provides lowerand upper bounds when considering a single optimization period, this might not be true for each individual optimization in a rolling horizon framework if the starting value for the stored energy level, carried over from the previous optimization period, differs. Therefore, they should be interpreted as lower- and upper bound approximations rather than true bounds when consecutively considering multiple optimization problems. The third stepwise function is centered along the piecewise linear function (Fig. 6), but does not provide information on whether the obtained result is an overor underestimation.

The objective function is (24), with $u \in \mathbb{U}$ the set of steps of the stepwise market resilience function, and $p_{h, u}^{\mathrm{c}}, p_{h, u}^{\mathrm{d}}, Y_{h, u}^{\mathrm{sw}}$ the charge power, discharge power, and DA price that correspond to step $u$, respectively. The objective function is still subject to (3) - (11) and (20), and now additionally subject to (25) - (30), with $X_{h, u}^{\mathrm{sw}, \mathrm{lo}}$ and $X_{h, u}^{\mathrm{sw}, u \mathrm{p}}$ being the lower and upper bound of the (dis)charge volume corresponding to step $u$, respectively. The binary variables $s_{h, u}$ correspond to the steps of the stepwise function, and can be considered as Special Ordered Set of type one (SOS1) variables [51]. The resulting problem is formulated as a MILP which is solved in GAMS using the CPLEX solver:

\footnotetext{
${ }^{9}$ Alternatively, an iterative approach could be applied in which first a rather large step size is used, after which it is successively refined in the neighborhood of the most recent solution.

${ }^{10}$ Less favorable indicates higher prices when charging and lower prices when discharging, while more favorable indicates lower prices when charging and higher prices when discharging.
} 


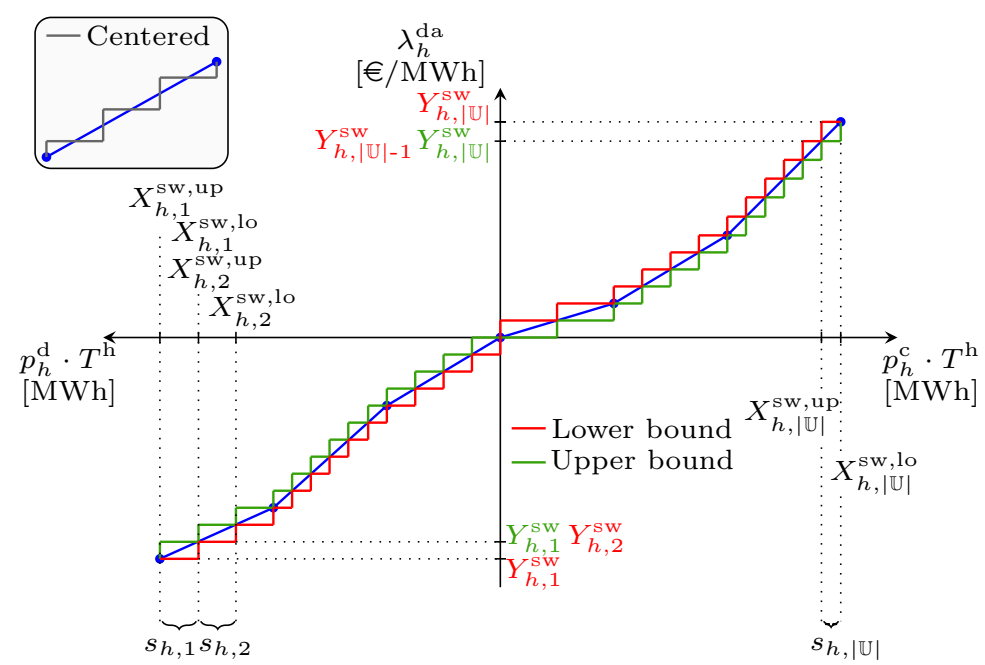

Figure 6: Lower bound, upper bound, and centered stepwise approximation of the piecewise linear market resilience functions.

$$
\begin{aligned}
& \pi^{\mathrm{op}}=\max _{\substack{b_{h}, c^{\mathrm{cyc}}, e_{h}, n^{\mathrm{cyc}}, p_{h}^{\mathrm{c}}, p_{h, u}^{\mathrm{c}}, p_{h}^{\mathrm{d}}, p_{h, u}^{\mathrm{d}}, s_{h, u}}} \sum_{h \in \mathbb{H}} \sum_{u \in \mathbb{U}}\left[Y_{h, u}^{\mathrm{sw}} \cdot\left[T^{\mathrm{h}} \cdot\left(p_{h, u}^{\mathrm{d}}-p_{h, u}^{\mathrm{c}}\right)\right]\right] /\left(|\mathbb{H}| \cdot T^{\mathrm{h}}\right)-c^{\mathrm{cyc}}, \\
& \text { s.t. }(3)-(11),(20) \text {, } \\
& \sum_{u \in \mathbb{U}} p_{h, u}^{\mathrm{c}}=p_{h}^{\mathrm{c}}, \quad \forall h \in \mathbb{H}, \\
& \sum_{u \in \mathbb{U}} p_{h, u}^{\mathrm{d}}=p_{h}^{\mathrm{d}}, \quad \forall h \in \mathbb{H}, \\
& s_{h, u} \cdot X_{h, u}^{\mathrm{sw}, \text { lo }} \leq T^{\mathrm{h}} \cdot\left(p_{h, u}^{\mathrm{d}}-p_{h, u}^{\mathrm{c}}\right) \leq s_{h, u} \cdot X_{h, u}^{\mathrm{sw}, \mathrm{up}}, \quad \forall h \in \mathbb{H}, u \in \mathbb{U}, \\
& \sum_{u \in \mathbb{U}} s_{h, u}=1, \quad \forall h \in \mathbb{H}, \\
& p_{h, u}^{\mathrm{c}}, p_{h, u}^{\mathrm{d}} \in \mathbb{R}_{+}, s_{h, u} \in\{0,1\}, \quad \forall h \in \mathbb{H}, u \in \mathbb{U}, \\
& \mathbb{U}=\left\{1,2, \ldots, \sum_{k \in \mathbb{K} \backslash\{|\mathbb{K}|\}} N_{h, k}^{\mathrm{sw}}\right\}, \quad \forall h \in \mathbb{H} .
\end{aligned}
$$

\section{$5 \quad$ Results}

Unless specified otherwise, the used storage plant characteristics, along with other input data, are displayed in Table 2, and serve to model typical PHS plants. In addition, Fig. 7 provides information on the average market resilience curve slope up to $500 \mathrm{MWh}$ additional supply and $500 \mathrm{MWh}$ additional demand.

\subsection{Computational performance and accuracy of the stepwise ap- proximation}

Table 3 validates the use of the stepwise approximations for the piecewise linear market resilience functions, in order to move from a nonconvex MIQP to a MILP. Both the 
Table 2: Table of input parameters.

\begin{tabular}{clclclcl}
\hline$C^{\text {fix }, \mathrm{e}}$ & $50 € / \mathrm{MWh}$ & $N^{\text {cyc }}$ & 100000 & $P^{\text {d,min }}$ & $0 \mathrm{MW}$ & $R^{\text {d,up }}$ & $50 \% / \min$ \\
$E^{\text {max }}$ & $2000 \mathrm{MWh}$ & $P^{\mathrm{c}, \max }$ & $500 \mathrm{MW}$ & $R^{\mathrm{c}, \mathrm{do}}$ & $50 \% / \mathrm{min}$ & $\eta^{\mathrm{c}}$ & $86.6 \%$ \\
$E^{\text {min }}$ & $0 \mathrm{MWh}$ & $P^{\mathrm{c}, \min }$ & $0 \mathrm{MW}$ & $R^{\text {c,up }}$ & $50 \% / \mathrm{min}$ & $\eta^{\text {d }}$ & $86.6 \%$ \\
$N^{\text {cal }}$ & 50 years & $P^{\text {d,max }}$ & $500 \mathrm{MW}$ & $R^{\text {d,do }}$ & $50 \% / \mathrm{min}$ & & \\
\hline$|\mathbb{H}|$ & 48 & $|\mathbb{K}|$ & 7 & $S^{\text {tar }}$ & $1.0 € / \mathrm{MWh}$ & $T^{\mathrm{h}}$ & $1 \mathrm{~h}$ \\
\hline
\end{tabular}

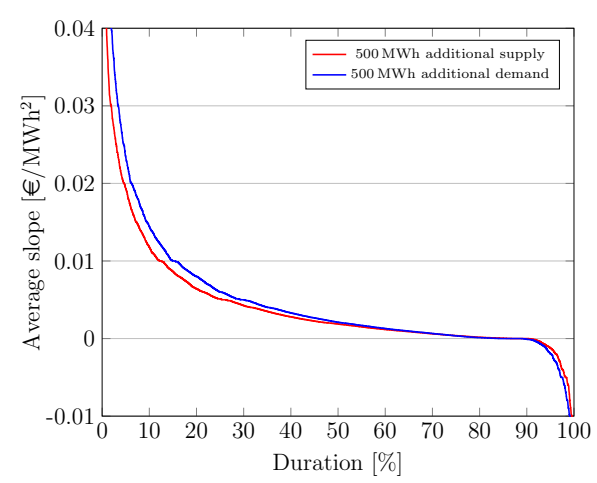

Figure 7: Average market resilience curve slope [€/MWh $\left.{ }^{2}\right]$ up to $500 \mathrm{MWh}$ additional demand (upward slope from the MRP) and up to $500 \mathrm{MWh}$ additional supply (downward slope from the MRP), Belgium, 2014. Positive values indicate intuitive price-effects, while negative values indicate counterintuitive price-effects.

operating profit $\pi^{\mathrm{op}}$ and computation time ${ }^{11}$ following the stepwise approximation are expressed in relative terms compared to the piecewise linear values (i.e., the values for the MIQP represent $100 \%$ ). This is done for twelve separate optimization periods of 12 time steps each, as for longer periods (e.g., 24 time steps) multiple hours of computation time did not suffice to solve the nonconvex MIQP to optimality.

Table 3 shows that $\pi^{\text {op }}$ following the stepwise approximation effectively provides tight lower- and upper bounds to $\pi^{\mathrm{op}}$ following the piecewise linear functions, while only requiring a fraction of the computation time. In addition, these bounds become more accurate as $S^{\text {tar }}$ is set to a smaller value while only incurring a slight increase in computation time, with the $S^{\text {tar }}=0.1 € / M W h$ bounds to $\pi^{\text {op }}$ located in between the $S^{\text {tar }}=1.0 € /$ MWh bounds. Although the centered stepwise approximation provides accurate estimations of $\pi^{\mathrm{op}}$ as well, these might be both under- or overestimations.

\subsection{Operating profit as a function of storage size}

Fig. 8 shows $\pi^{\text {op }}$ for 2014 for increasing storage power rating sizes assuming a fixed discharge energy-to-power ratio $E^{\max } / P^{\mathrm{d}, \max }$ of $4 \mathrm{~h}$. Fig. 8a displays $\pi^{\mathrm{op}}$ in absolute values, while Fig. $8 \mathrm{~b}$ illustrates $\pi^{\mathrm{op}}$ relative to the expected operating profit when assuming to be a price-taker in the market. When the storage operator assumes to be a price-taker and thus not considers the price-effect when deciding on the (dis)charge schedule, the expected $\pi^{\text {op }}$ increases linearly with the storage size, as price spreads remain constant. However, when ex-post calculating the resulting DA price using the market resilience data, the realized $\pi^{\text {op }}$ given the (dis)charge schedule decided upon by the price-taking storage operator is lower, with the gap between expected and realized

\footnotetext{
${ }^{11}$ The computation time is defined as the time used by the solver, given by the GAMS parameter "resusd", while all models are solved to optimality by setting the GAMS option "optcr" to zero.
} 
Table 3: Validation of the stepwise approximation's lower- and upper bound to $\pi^{\text {op }}$ following piecewise linear price-effects, as well as of the centered stepwise approximation, Belgium, 2014 The MILP values are shown relative to the MIQP values, which represent $100 \%$.

\begin{tabular}{|c|c|c|c|c|c|c|c|c|c|c|c|c|c|}
\hline \multirow{3}{*}{\multicolumn{2}{|c|}{$\begin{array}{l}\text { Optimization } \\
\text { period }\end{array}$}} & \multicolumn{6}{|c|}{$S^{\text {tar }}=0.1 € / \mathrm{MWh}$} & \multicolumn{6}{|c|}{$S^{\text {tar }}=1.0 € / \mathrm{MWh}$} \\
\hline & & \multicolumn{2}{|c|}{ Lower bound } & \multicolumn{2}{|c|}{ Upper bound } & \multicolumn{2}{|c|}{ Centered } & \multicolumn{2}{|c|}{ Lower bound } & \multicolumn{2}{|c|}{ Upper bound } & \multicolumn{2}{|c|}{ Centered } \\
\hline & & $\pi^{o p}$ & Time & $\pi^{o p}$ & Time & $\pi^{o p}$ & Time & $\pi^{o p}$ & Time & $\pi^{o p}$ & Time & $\pi^{o p}$ & Time \\
\hline Hour & Date & [\%] & {$[\%]$} & [\%] & [\%] & [\%] & [\%] & [\%] & {$[\%]$} & [\%] & {$[\%]$} & [\%] & {$[\%]$} \\
\hline $1-12$ & Jan 1 & 99.92 & 1.69 & 100.60 & 1.82 & 100.26 & 1.67 & 99.92 & 0.15 & 102.46 & 0.17 & 101.18 & 0.19 \\
\hline $1-12$ & Feb 1 & 99.98 & 0.03 & 100.13 & 0.03 & 100.06 & 0.03 & 99.96 & 0.01 & 101.16 & 0.01 & 100.56 & 0.01 \\
\hline $1-12$ & Mar 1 & 99.92 & 0.27 & 100.05 & 0.26 & 99.97 & 0.24 & 99.67 & 0.02 & 100.46 & 0.03 & 100.04 & 0.02 \\
\hline $1-12$ & Apr 1 & 99.95 & 18.43 & 101.16 & 17.75 & 100.55 & 19.78 & 99.89 & 1.80 & 105.91 & 1.57 & 102.89 & 1.80 \\
\hline $1-12$ & May 1 & 99.93 & 0.38 & 100.16 & 0.43 & 100.04 & 0.42 & 99.72 & 0.04 & 100.90 & 0.03 & 100.31 & 0.04 \\
\hline $1-12$ & Jun 1 & 99.90 & 0.76 & 100.06 & 0.89 & 99.98 & 0.85 & 99.16 & 0.05 & 100.16 & 0.05 & 99.66 & 0.05 \\
\hline $1-12$ & Jul 1 & 99.94 & 0.39 & 100.15 & 0.42 & 100.05 & 0.40 & 98.78 & 0.01 & 100.55 & 0.02 & 99.67 & 0.01 \\
\hline $1-12$ & Aug 1 & 99.99 & 0.91 & 100.53 & 1.00 & 100.26 & 0.99 & 99.67 & 0.08 & 103.71 & 0.10 & 101.49 & 0.12 \\
\hline $1-12$ & Sep 1 & 99.97 & 5.61 & 100.33 & 6.51 & 100.15 & 6.5 & 98.84 & 0.49 & 101.92 & 0.49 & 100.30 & 0.69 \\
\hline $1-12$ & Oct 1 & 100.00 & 0.05 & 100.36 & 0.04 & 100.18 & 0.05 & 99.93 & 0.01 & 102.68 & 0.01 & 101.27 & 0.01 \\
\hline $1-12$ & Nov 1 & 99.86 & 0.61 & 100.15 & 0.58 & 100.01 & 0.62 & 98.78 & 0.07 & 100.92 & 0.05 & 99.85 & 0.05 \\
\hline $1-12$ & Dec 1 & 99.70 & 0.18 & 100.20 & 0.18 & 99.95 & 0.29 & 98.34 & 0.01 & 101.27 & 0.01 & 99.66 & 0.01 \\
\hline
\end{tabular}

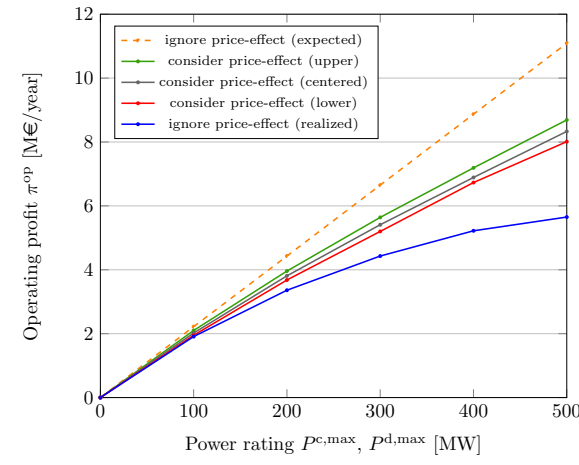

(a) Absolute values.

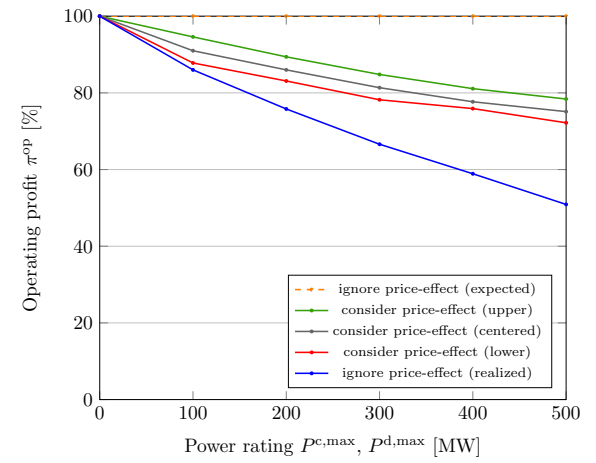

(b) Relative values.

Figure 8: Expected and realized $\pi^{\text {op }}$ when not considering the price-effect, and a lower- and upper bound, as well as a centered, stepwise approximation to $\pi^{\mathrm{op}}$ when considering the piecewise linear price-effect, Belgium, 2014.

$\pi^{\text {op }}$ being negligible for small storage plants but increasing with the storage size. Contrarily, in order to retrieve the upper limit to $\pi^{\text {op }}$ of additional storage capacity, the price-effect is already considered at the decision stage. The results show both a lower- and upper bound approximation to $\pi^{\mathrm{op}}$ following the piecewise linear priceeffect, as well as an approximation based on a centered stepwise function. Nevertheless, as more capacity is used for arbitrage the incremental $\pi^{\mathrm{op}}$ decreases both in the pricetaker and price-maker approach due to the price-effect, resulting in a trade-off between the capacity used and the average profit per unit.

\section{3 (Dis)charge schedule and price profile}

A duration curve of the (dis)charge actions for 2014 is displayed in Fig. 9, for both a storage operator which is assumed to be a price-taker in the market and a storage operator that takes into account its price-effect. In the former case the (dis)charge power rating will always be used to its full capacity when (dis)charging, unless bounded by the limited energy storage capacity. Contrarily, in the latter case fewer full load hours (19\% vs. $31.6 \%)$ are observed when taking into account the price-effect of (dis)charge actions to keep price spreads from diminishing a lot. Although (dis)charge actions are partially shifted to neighboring hours, observed through the increased number of op- 


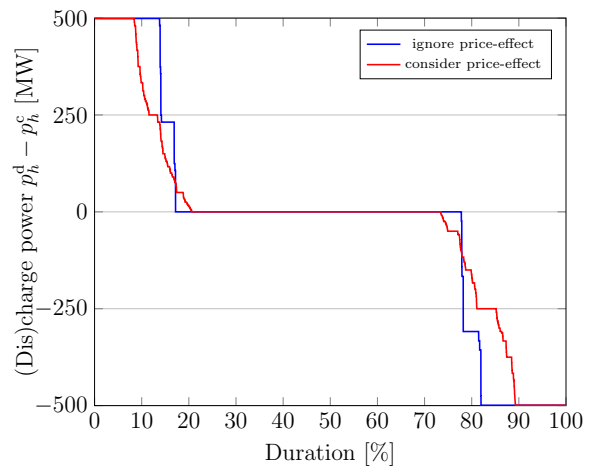

Figure 9: Considering the price-effect leads to fewer full load hours, more operational hours, and less (dis)charged energy in total, Belgium, 2014. The illustrated (dis)charge actions for when considering the price-effect are those of the lower bound approximation.

erational hours of the storage plant ( $47.3 \%$ vs. $39.6 \%$ ), in total less energy is charged (733.2 GWh vs. 893.3 GWh) and discharged (549.9 GWh vs. $670 \mathrm{GWh}$ ).

Fig. 10 shows an example of a daily price profile and (dis)charge schedule. It shows that when not considering the price-effect the storage operator (dis)charges at full power rating, while when considering the price-effect a trade-off occurs between used capacity and remaining price-spread. The charge action is partly shifted to market period 4, which is not just characterized by a smaller price-effect compared to market periods 5 to 8 , but by a counterintuitive one as the price decreases when charging. The resilience function for market period 4 shows a slightly decreasing slope up to $250 \mathrm{MWh}$ additional demand, after which the slope increases, hence a charge action of $250 \mathrm{MW}$ during hour 4.

\subsection{Discussion: yearly arbitrage value compared to annualized in- vestment cost}

To provide some perspective on the order of magnitude of the obtained DA arbitrage value for Belgium for 2014, it is compared to the annualized investment cost of the considered PHS plant. While the available literature provides a wide range of investment cost estimations (e.g., [53]), this article assumes a cost of $50 € / \mathrm{kWh}$ for the energy-component and $750 € / \mathrm{kW}$ for the power-component. Given an assumed weighted average cost of capital of $5 \%$, the annualized investment cost is $2.7 € / \mathrm{kWh}$ and $41.1 € / \mathrm{kW}$. For a storage plant with the characteristics displayed in Table 2, the total annualized investment cost is $25.95 \mathrm{M} €$. Even when considering the upper limit to $\pi^{\text {op }}$, i.e., assuming perfect foresight of both prices and price-effects, in 2014 DA arbitrage only accounts for $30.9 \%$ (lower bound approximation) to $33.5 \%$ (upper bound approximation) of the annualized investment cost.

\section{Conclusions}

Although the value of electricity storage arbitrage is directly related to the frequency and size of price spreads, it is also a function of the price-effect of (dis)charge actions. The price-effect represents the degree to which additional demand increases off-peak prices and additional supply decreases on-peak prices, and is, ceteris paribus, inversely correlated to the arbitrage value. While the impact of the price-effect is negligible for small storage volumes, it reduces the arbitrage value significantly for large storage volumes. In this article the price-effect is taken into account by considering real-world 


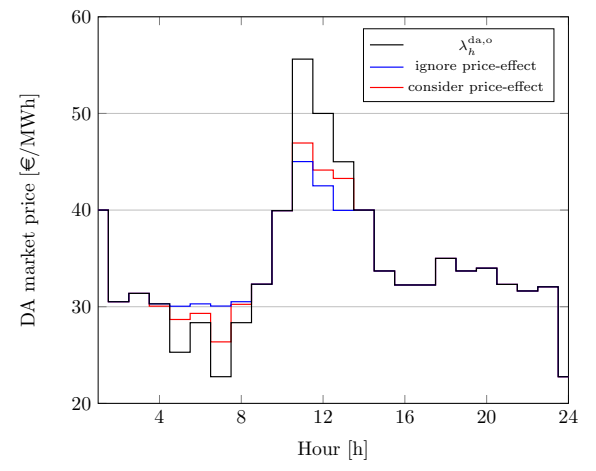

(a) Price profile.

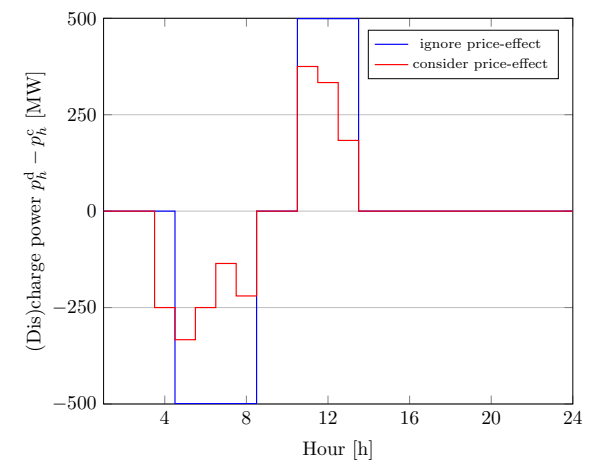

(b) (Dis)charge schedule.

Figure 10: Illustration of the price profile and the (dis)charge schedule. When not considering the price-effect the storage operator (dis)charges at full power rating, while when considering the price-effect a trade-off emerges between used (dis)charge power and remaining price-spread, Belgium, May 10 2014. The illustrated price profile and (dis)charge schedule for when considering the price-effect are those of the lower bound approximation.

market resilience data, available in the form of hourly piecewise linear functions, and published by multiple European power exchanges. Since this data is only available expost, their application mainly lies in estimating the upper limit to the arbitrage value of additional storage capacity in a certain market given current market conditions, and the evaluation of the performance of the price-taking assumption and price-making assumptions based on more conceptual and simplified price-effects. The former assumes that the price-effect is already taken into account at the (dis)charge decision stage, while the latter can be done by ex-post calculating the realized profit as opposed to the anticipated profit.

Future work includes three topics. First, the analysis of the price-effect in the settlement side of the real-time balancing market, as in this market price spreads are much larger compared to the DA market, but the price-effect is significantly larger as well because of the smaller market size. Second, the estimation of a lower limit to the arbitrage value under uncertainty in addition to the upper limit, which will allow to provide an operating profit range under imperfect foresight. Third, since the results show that using more capacity to arbitrage price spreads decreases the value per unit, maximizing the value of storage will likely require the aggregation of multiple applications. The value of individual applications cannot simply be added as use for one application may interfere with another, therefore it is necessary to co-optimize the applications.

\section{Acknowledgment}

The authors would like to thank Cedric De Jonghe and Anya Castillo for valuable comments, and Research Foundation Flanders (FWO) for providing Tom Brijs with a travel grant for an extended research visit at The Johns Hopkins University. The authors would also like to thank the Department of Energy, Water, and Transportation at DIW Berlin for hosting DIW Research Fellow Sauleh Siddiqui and Tom Brijs for discussing this research in October 2015. 


\section{References}

[1] F. Geth, T. Brijs, J. Kathan, J. Driesen, R. Belmans, An overview of largescale stationary electricity storage plants in Europe: Current status and new developments, Renew. \& Sustainable Energy Rev. 52 (2015) 1212-1227. doi: $10.1016 / j$.rser. 2015.07.145

[2] E. Lannoye, D. Flynn, M. O'Malley, Evaluation of power system flexibility, IEEE Trans. Power Syst. 27 (2) (2012) 922-931. doi:10.1109/TPWRS.2011.2177280.

[3] E. Lannoye, D. Flynn, M. O’Malley, Transmission, variable generation, and power system flexibility, IEEE Trans. Power Syst. 30 (1) (2015) 57-66. doi:10.1109/ TPWRS. 2014.2321793.

[4] A. Castillo, D. F. Gayme, Grid-scale energy storage applications in renewable energy integration: A survey, Energy Convers. and Management 87 (2014) 885-894. doi:10.1016/j.enconman.2014.07.063.

[5] A. Akhil, G. Huff, A. Currier, B. Kaun, D. Rastler, S. B. Chen, A. Cotter, D. Bradshaw, W. Gauntlett, DOE / EPRI 2013 Electricity Storage Handbook in Collaboration with NRECA, Tech. Rep. SAND2013-5131, Sandia National Laboratories, Albuquerque, New Mexico and Livermore, California (2013). URL http://www.sandia.gov/ess/publications/SAND2013-5131.pdf

[6] X. Xi, R. Sioshansi, V. Marano, A stochastic dynamic programming model for co-optimization of distributed energy storage, Energy Syst. 5 (3) (2013) 475-505. doi:10.1007/s12667-013-0100-6.

[7] H. Pandžić, Y. Wang, T. Qiu, Y. Dvorkin, D. S. Kirschen, Near-optimal method for siting and sizing of distributed storage in a transmission network, IEEE Trans. Power Syst. 30 (5) (2015) 2288-2300. doi:10.1109/TPWRS.2014.2364257.

[8] M. Shahidehpour, H. Yamin, Z. Li, Market Operations in Electric Power Systems: Forecasting, Scheduling, and Risk Management, IEEE-Wiley, New York, USA, 2002.

[9] T. Li, M. Shahidehpour, Price-based unit commitment : A case of lagrangian relaxation versus mixed integer programming, IEEE Trans. Power Syst. 20 (4) (2005) 2015-2025. doi:10.1109/TPWRS.2005.857391.

[10] A. D. Lamont, Assessing the economic value and optimal structure of large-scale electricity storage, IEEE Trans. Power Syst. 28 (2) (2013) 911-921. doi:10.1109/ TPWRS . 2012.2218135

[11] W. P. Schill, C. Kemfert, Modeling strategic electricity storage: the case of pumped hydro storage in Germany, The Energy J. 32 (3) (2011) 59-87. doi: 10.5547/ISSN0195-6574-EJ-Vo132-No3-3.

[12] A. S. A. Awad, J. D. Fuller, T. H. M. EL-Fouly, M. M. A. Salama, Impact of energy storage systems on electricity market equilibrium, IEEE Trans. Sustainable Energy 5 (3) (2014) 875-885. doi:10.1109/TSTE.2014.2309661.

[13] C. Suazo-Martínez, E. Pereira-Bonvallet, R. Palma-Behnke, X. P. Zhang, Impacts of energy storage on short term operation planning under centralized spot markets, IEEE Trans. Smart Grid 5 (2) (2014) 1110-1118. doi:10.1109/TSG. 2013. 2281828.

[14] B. Nyamdash, E. Denny, The impact of electricity storage on wholesale electricity prices, Energy Policy 58 (2013) 6-16. doi:10.1016/j.enpol.2012.11.033.

[15] R. Lueken, J. Apt, The effects of bulk electricity storage on the PJM market, Energy Syst. 5 (4) (2014) 677-704. doi:10.1007/s12667-014-0123-7. 
[16] R. Sioshansi, P. Denholm, T. Jenkin, J. Weiss, Estimating the value of electricity storage in PJM: Arbitrage and some welfare effects, Energy Economics 31 (2) (2009) 269-277. doi:10.1016/j .eneco.2008.10.005.

[17] G. Steeger, L. A. Barroso, S. Rebennack, Optimal bidding strategies for hydroelectric producers: A literature survey, IEEE Trans. Power Syst. 29 (4) (2014) 1758-1766. doi:10.1109/TPWRS.2013.2296400.

[18] F. C. Figueiredo, P. C. Flynn, E. A. Cabral, The economics of energy storage in 14 deregulated power markets, Energy Stud. Rev. 14 (2) (2006) 131-152. doi: 10.15173/esr.v14i2.494.

[19] C. K. Ekman, S. H. Jensen, Prospects for large scale electricity storage in Denmark, Energy Convers. and Management 51 (6) (2010) 1140-1147. doi: 10.1016/j.enconman.2009.12.023.

[20] P. Denholm, R. Sioshansi, The value of compressed air energy storage with wind in transmission-constrained electric power systems, Energy Policy 37 (8) (2009) 3149-3158. doi:10.1016/j.enpol.2009.04.002.

[21] F. Graves, T. Jenkin, D. Murphy, Opportunities for electricity storage in deregulating markets, The Electricity J. 12 (8) (1999) 46-56. doi:10.1016/ S1040-6190 (99) 00071-8.

[22] G. Bathurst, G. Strbac, Value of combining energy storage and wind in shortterm energy and balancing markets, Electr. Power Syst. Res. 67 (1) (2003) 1-8. doi:10.1016/S0378-7796(03)00050-6.

[23] E. Barbour, I. A. G. Wilson, I. G. Bryden, P. G. McGregor, P. A. Mulheran, P. J. Hall, Towards an objective method to compare energy storage technologies: development and validation of a model to determine the upper boundary of revenue available from electrical price arbitrage, Energy \& Environ. Science 5 (1) (2012) 5425. doi:10.1039/c2ee02419e.

[24] H. Khani, M. R. D. Zadeh, Online adaptive real-time optimal dispatch of privately owned energy storage systems using public-domain electricity market prices, IEEE Trans. Power Syst. 30 (2) (2015) 930-938. doi:10.1109/TPWRS.2014. 2336753.

[25] D. Connolly, H. Lund, P. Finn, B. V. Mathiesen, M. Leahy, Practical operation strategies for pumped hydroelectric energy storage (PHES) utilising electricity price arbitrage, Energy Policy 39 (7) (2011) 4189-4196. doi:10.1016/j.enpol. 2011.04 .032$.

[26] R. Sioshansi, P. Denholm, T. Jenkin, A comparative analysis of the value of pure and hybrid electricity storage, Energy Economics 33 (1) (2011) 56-66. doi: $10.1016 / j$. eneco.2010.06.004.

[27] H. Lund, G. Salgi, B. Elmegaard, A. N. Andersen, Optimal operation strategies of compressed air energy storage (CAES) on electricity spot markets with fluctuating prices, Appl. Therm. Engineering 29 (5-6) (2009) 799-806. doi: 10.1016/j . applthermaleng.2008.05.020.

[28] R. Walawalkar, J. Apt, R. Mancini, Economics of electric energy storage for energy arbitrage and regulation in New York, Energy Policy 35 (4) (2007) 2558-2568. doi:10.1016/j.enpol.2006.09.005.

[29] E. Delarue, P. Van Den Bosch, W. D'haeseleer, Effect of the accuracy of price forecasting on profit in a Price Based Unit Commitment, Electr. Power Syst. Res. 80 (10) (2010) 1306-1313. doi:10.1016/j.epsr.2010.05.001.

[30] H. Mohsenian-Rad, Optimal bidding, scheduling, and deployment of battery systems in California day-ahead energy market, IEEE Trans. Power Syst. 31 (1) (2015) 442-453. doi:10.1109/TPWRS.2015.2394355. 
[31] R. Sioshansi, Welfare impacts of electricity storage and the implications of ownership, The Energy J. 31 (2) (2009) 173-198. doi:10.5547/ ISSN0195-6574-EJ-Vol31-No2-7.

[32] X. He, E. Delarue, W. D'haeseleer, J.-M. Glachant, A novel business model for aggregating the values of electricity storage, Energy Policy 39 (3) (2011) 1575-1585. doi:10.1016/j.enpol.2010.12.033.

[33] J. A. Sousa, F. Teixeira, S. Faias, Impact of a price-maker pumped storage hydro unit on the integration of wind energy in power systems, Energy 69 (2014) 3-11. doi:10.1016/j.energy.2014.03.039.

[34] C. G. Baslis, A. G. Bakirtzis, Mid-term stochastic scheduling of a price-maker hydro producer with pumped storage, IEEE Trans. Power Syst. 26 (4) (2011) 1856-1865. doi:10.1109/TPWRS.2011.2119335.

[35] D. S. Kirschen, G. Strbac, P. Cumperayot, D. de Paiva Mendes, Factoring the elasticity of demand in electricity prices, IEEE Trans. Power Syst. 15 (2) (2000) 612-617. doi:10.1109/59.867149.

[36] N. Lu, J. H. Chow, A. A. Desrochers, Pumped-storage hydro-turbine bidding strategies in a competitive electricity market, IEEE Trans. Power Syst. 19 (2) (2004) 834-841. doi:10.1109/TPWRS.2004.825911.

[37] B. F. Hobbs, Linear complementarity models of Nash-Cournot competition in bilateral and POOLCO power markets, IEEE Trans. Power Syst. 16 (2) (2001) 194-202. doi:10.1109/59.918286.

[38] B. F. Hobbs, F. A. M. Rijkers, Strategic generation with conjectured transmission price responses in a mixed transmission pricing system - Part 1: Formulation, IEEE Trans. Power Syst. 19 (2) (2004) 707-717. doi:10.1109/TPWRS. 2003. 821628.

[39] P. D. Klemperer, M. A. Meyer, Supply function equilibria in oligopoly under uncertainty, Econometrica 57 (6) (1989) 1243-1277.

[40] H. M. I. Pousinho, J. Contreras, J. P. S. Catalão, Operations planning of a hydro producer acting as a price-maker in an electricity market, in: IEEE PES General Meeting, San Diego, USA, 2012, pp. 1-7. doi:10.1109/PESGM.2012.6343941.

[41] H. M. I. Pousinho, J. Contreras, A. G. Bakirtzis, J. P. S. Catalão, Riskconstrained scheduling and offering strategies of a price-maker hydro producer under uncertainty, IEEE Trans. Power Syst. 28 (2) (2013) 1879-1887. doi: 10.1109/TPWRS. 2012.2229473.

[42] A. Baíllo, M. Ventosa, M. Rivier, A. Ramos, Optimal Offering Strategies for Generation Companies Operating in Electricity Spot Markets, IEEE Trans. Power Syst. 19 (2) (2004) 745-753. doi:10.1109/TPWRS.2003.821429.

[43] Belpex, Historical Data (2016). URL http://www.belpex.be/market-results/historical-data/

[44] APX, Market results - Dashboard (2016). URL https://www . apxgroup.com/market-results/apx-power-nl/dashboard/

[45] EPEXSPOT, Downloads - Market data (2016). URL https://www.epexspot.com/en/extras/download-center/market_data

[46] P. N. Biskas, D. I. Chatzigiannis, A. G. Bakirtzis, European electricity market integration with mixed market designs-part I: Formulation, IEEE Trans. Power Syst. 29 (1) (2014) 458-465. doi:10.1109/TPWRS.2013.2245923.

[47] M. Ventosa, A. Baíllo, A. Ramos, M. Rivier, Electricity market modeling trends, Energy Policy 33 (7) (2005) 897-913. doi:10.1016/j.enpol.2003.10.013. 
[48] H. Varian, Microeconomic Analysis, 3rd Edition, W.W. Norton \& Company, New York, USA, 1992.

[49] J. Tant, F. Geth, D. Six, P. Tant, J. Driesen, Multiobjective battery storage to improve PV integration in residential distribution grids, IEEE Trans. Sustainable Energy 4 (1) (2013) 182-191. doi:10.1109/TSTE.2012.2211387.

[50] CPLEX, ILOG CPLEX optimizer (2016).

URL http://www.ilog.com/products/cplex

[51] E. M. L. Beale, J. A. Tomlin, Special facilities in a general mathematical programming system for non-convex problems using order sets of variables, in: J. Lawrence (Ed.), Fifth International Conference on Operational Research, Tavistock Publishing, London, 1970, pp. 447-454.

[52] M. R. Bussieck, A. S. Drud, SBB: A new solver for mixed integer nonlinear programming (2001).

URL http://www.gams.com/presentations/or01/sbb.pdf

[53] H. L. Ferreira, R. Garde, G. Fulli, W. Kling, J. P. Lopes, Characterisation of electrical energy storage technologies, Energy 53 (2013) 288-298. doi:10.1016/ j.energy. 2013.02.037. 